\title{
Accelerations in water waves by extended Particle Image Velocimetry
}

by

\author{
Atle Jensen, J. Kristian Sveen, Didier Clamond and John Grue \\ Mechanics Division, Department of Mathematics, \\ University of Oslo, Norway
}

Jean-Baptiste Richon and Callum Gray

Optical Flow Systems, Scotland

Particle Image Velocimetry (PIV) measures instantaneous velocity across an extended area of flow by recording the motion of tracers suspended in and moving with the fluid. This principle is extended to the measurement of higher moments of the velocity field (acceleration) by recording the velocity field at two separate time instants using two cameras, viewing the same region of flow. Planar illumination of large areas within a hydrodynamic flow is achieved using a scanned argon ion laser beam and individual velocity measurements are made by cross-correlating image pairs acquired with a cooled, frame straddling camera. A high speed acousto-optic modulator is used to shut off the $C W$ laser after two scans of the flow have been captured by the first camera. The modulator switches the beam back on for the second velocity measurement after a programmed delay. Synchronization of the cameras and beam modulator with the scanning beam system is achieved with a purpose built multi-channel synchronizer device and operated from an integrated modular tree-based acquisition and processing software system.

The extended PIV system is employed to measure the velocities and accelerations in periodic waves in a precise laboratory wave tank. A complementary theoretical description of Stokes waves provides a comparison with the measurements. The theoretical model is very precise, with an error term being less than $0.5 \%$ relative to the primary wave for the conditions of the experiments. The purpose is to test the measurement system and to judge the accuracy of the wave experiments under realistic and controllable conditions in the laboratory. Good agreement between the experiments and theory is found. The relative accuracy of the present experiments and measurements may be quantified in terms of the standard deviation due to an ensemble of measurements. In the best case we find a relative standard deviation of $0.6 \%$ for the velocity measurements and $2 \%$ for the accelerations. It is indicated that such an accuracy may be generally achieved by appropriately choosing the size of the field of view.

\section{Introduction}

The rapid development of computers and advanced experimental tools has made it possible to perform investigations one earlier only could dream of. Today, almost every fluid mechanisist employs computers to complement theoretical studies and to analyze the equations of fluid flow for an increasingly wide range of applications. Less attention is perhaps paid to the considerable potential of an interplay between theoretical modelling and precise laboratory experiments. The recent advances of experimental techniques like particle image 
velocimetry (PIV) provide, however, both desired and attractive supplements to theoretical and computational investigations of complex problems in fluid mechanics. Presice experiments and advanced computation models developed in parallel may contribute to a better understanding of the physical problem and to a more robust modelling of the phenomenon under consideration. Precise experiments may also provide understanding of the inherent physics of new problems where simulation models are not successful. In the present paper we shall describe a particle imaging system designed with the purpose to complement theoretical analysis. More precisely, we shall describe an extended particle imaging system which can be used to measure not only the velocity field of the fluid, but also the accelerations. The ultimate goal has been to develop a system giving measurements, with sufficiently high precision that could be used as input to theoretical modelling of various complex flows.

The acceleration field is calculated from the difference between two consecutive velocity fields. The latter are measured using directionally resolved Digital Particle Image Velocimetry (DPIV). The system uses two separate cameras viewing the same region of flow to acquire PIV images with no limitation on the time between individual velocity measurements. Both cameras record the same field of view with a small angle between their respective viewing axes. Precise alignment between the two fields is achieved using cross-correlation analysis similar to that used to extract the velocity field from image pairs acquired from individual cameras. Alternatively one can use a dual camera system with beam combining optics (or a multi CCD array camera), but the technical setup outlined in this paper is in general more flexible as it is easily extendable to 3 -D PIV measurements and combined PIV and Laser Induced Fluorescence (LIF) measurements.

The acceleration measurement system is applied to water waves in a two-dimensional wave tank. The system is designed to cope with large measurement areas, typically up to $50 \mathrm{~cm}$ wide and high. To achieve this, a Continuous Wave argon ion laser coupled to a scanning beam system is used for illumination: this arrangement is particularly well suited to providing a large light sheet of uniform intensity. In addition, an acousto-optical cell modulates the light sheet so only one laser scan is recorded on each image acquired by the CCD cameras. Synchronization between the system components is achieved with a programmable multi-channel synchronizer. Overall configuration, control, acquisition and analysis are performed from PIV software incorporating an integrated data management and logging system.

For the validation of our method, we measure accelerations in incoming Stokes waves in both deep water and finite depth. Waves are generated by a piston type wave maker at one end of the tank and are absorbed by a beach at the other end. The measurements are performed for periodic progressive waves with wave slope up to 0.16 . The measurements are compared with Stokes wave theory in deep water and for finite water depth. We generally find excellent agreement between experiments and theory. In the case of deep water, the theoretical model is valid up to the third order in the wave slope, giving an error term being less than $0.5 \%$ relative to the primary wave. The theory is thus very precise. Previous measurements of acceleration in water waves have been performed by Jacobsen, Dewhirst and Greated (1997) and Chang and Liu (1998) and we briefly compare our measurements 
with their.

This paper is organized as follows. Section 2 describes the technical aspects of the system. The experiments are described in section 3 together with the wave theory. Section 4 shows results compared with theory, while finally section 5 contains a conclusion.

\section{Technical description of the PIV system}

The acceleration measurements presented here are based on pairs of PIV measurements, and thus measures the change in velocity over a known time period. The system uses two separate cameras, each viewing the same region of flow and each recording an image pair for PIV evaluation. The acceleration field is calculated from the difference between two consecutive velocity fields. We use a standard (digital) cross-correlation PIV technique. The system consists of an illumination part, two cameras, a synchronizer and PC software.

\subsection{Illumination}

Following the need for two PIV measurements, a minimum of four light pulses are required for our acceleration measurements. One method for generating these pulses is to use a scanning beam system as described by Gray et al. (1991) and shown in figure 1. In this case a continuous wave (CW) laser beam is collimated or slightly converging. This is deflected from an octagonal mirror to create a beam that repeatedly scans through 90 degrees. A parabolic mirror deflects and collimates the divergent pseudo light sheet into the flow area. This gives a stroboscopically pulsing light sheet that may be further modulated by shuttering the main $\mathrm{CW}$ beam with an acousto-optic modulator (AOM). The AOM is used to select two or more pulses for PIV recording. The light sheet formed with a $\mathrm{CW}$ laser and scanner is of very uniform intensity and well suited to PIV over large areas. The laser source is a Coherent Innova 300 argon ion laser emitting at all lines (488 - 514nm), with a maximum continuous power output of $10 \mathrm{~W}$. The beam is passed through an AOM crystal prior to being launched into a multimode optical fiber for delivery across the lab to the scanner positioned below the glass bed of the wave flume. The AOM crystal is driven by an RF amplifier which it is switched on and off by a logic input. In the off state, the beam is transmitted directly through the AOM with very little attenuation of intensity (less than 4\%). In the on state, the crystal medium is excited by a piezo-transducer; it then behaves like a diffraction grating. As the beam goes through the crystal, it is deviated by a known angle, which is a constant of the AOM crystal design. The launch optics of the fiberoptic link is positioned in such a way that the beam can be transmitted through the fiber only when it is deviated through the AOM. The combination of the AOM and the positioned fiber launch optics acts as a beam modulator with a response time of less than $1 \mu \mathrm{s}$. The output of the fiberoptic link is connected to the scanner via a lockable collet. The collimated beam is passed through a telescope arrangement that provides adjustment of the light sheet thickness by varying the position of the beam waist. The output of the scanner is a $50 \mathrm{~cm}$ wide pseudo light sheet. 
We note that the most common laser choice for PIV is a double cavity Nd:YAG with beam combining optics. The main benefit of this system is the flexibility of the pulse separations afforded by the use of two independent cavities and independence of pulse energy from pulse separation (compared with CW laser PIV). The main problem with the pulsed laser arrangement in the general case of acceleration measurement however, is the need for a minimum of four pulses per measurement. This requires four laser cavities and beam combining optics for four beams. This kind of system does exist but is extremely expensive. Additionally, light sheets formed with cylindrical optics will tend to be nonuniform in thickness and intensity especially when used over a large measurement area.
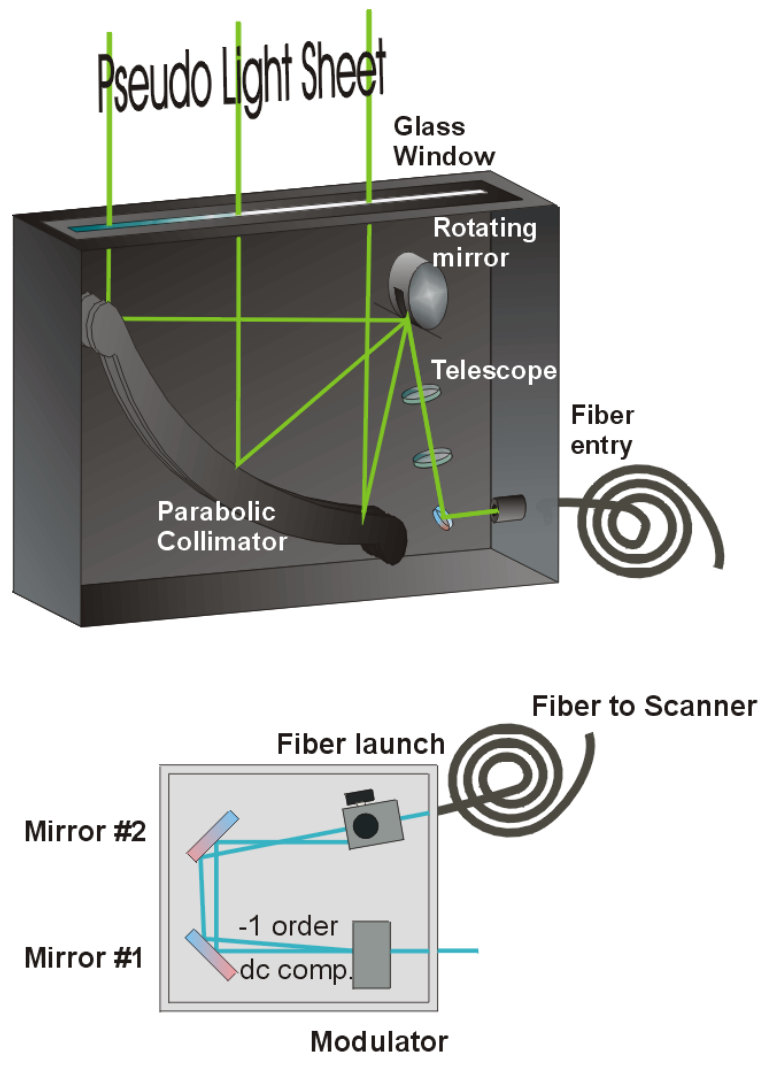

Figure 1: The scanning beam system and acousto-optical modulator (AOM).

\subsection{Camera system}

The system used here is an extension of a standard PIV configuration, using two separate cameras pointing to the same field of view with a small angle between them. The organization of its main components is shown in figure 2.

The two cameras used for image acquisition are high-sensitivity PCO Sensicam CCD cameras with a resolution of $1280 \times 1024$ pixels, and 12-bit digital outputs. To minimize 


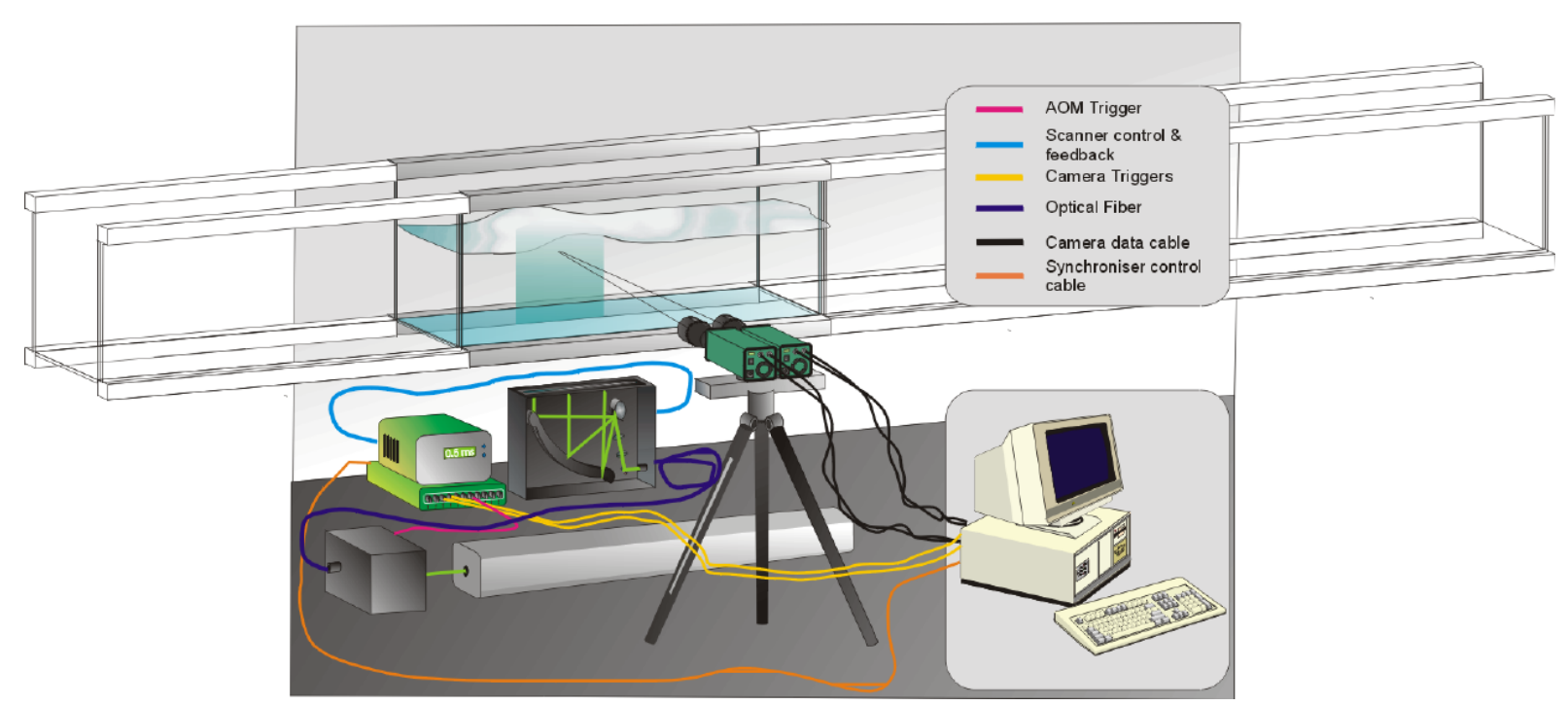

Figure 2: Schematic drawing of experimental setup.

noise and take full advantage of the available 12-bit dynamic range, the interline transfer $\mathrm{CCD}$ sensors are cooled to $-15^{\circ} \mathrm{C}$ and have a relatively low pixel readout rate. Images are transmitted to a host $\mathrm{PC}$ for storage and processing via high-speed digital fiberoptic link and a PCI frame grabber.

The cameras are used in dual image mode: on reception of a control trigger, a first frame is exposed, then stored in on-chip registers and read out while a second image is being exposed. With this mode of operation, very short inter-image times, down to 200ns, can be achieved when capturing an image pair. The "frame straddling" technique takes direct advantage of this feature to acquire PIV image pairs.

One characteristic of operating the cameras in dual image mode is that the exposure time of the first frame is controllable. When a scanning beam system is used this first exposure can be accurately controlled so that the time and duration of the first frame records a single scan.

The exposure time of the second frame is fixed, however. This is determined by the CCD readout time of approximately $100 \mathrm{~ms}$. This fixed length exposure would record more than one scan depending on the scan rate used. This limitation is overcome by gating the laser beam with an AOM so that the beam is switched off after the first scan of the second frame.

The beam is then switched on again for the subsequent exposure of images on the second camera, implying that these exposures may also be contained in the second image of the first camera (depending on the time separations used). The main effect of this double exposure is that bright areas of the field of view tend to be brighter on the double exposed image, thus leading in some cases to an increased number of false velocity vectors in these areas. 


\subsubsection{Synchronization}

In an image acquisition sequence consecutive scans of the flow field are recorded onto two consecutive frames of a frame straddling CCD camera. This requires each camera to be externally trigged. The delay and duration of the trigger control pulse determines the delay and duration of the image 1 exposure on each camera. The camera control pulses are generated by the system synchronizer, which receives a synchronization pulse from the scanner at every scan of the flow field (see system diagram). The synchronizer measures the frequency and phase of the sync. signal coming from the scanner and from this timebase generates trigger pulses for camera 1, camera 2 and the acousto-optic modulator. A timing diagram describing the temporal relationship between the scanner sync. signal, the camera and AOM triggers is given in figure 3 .

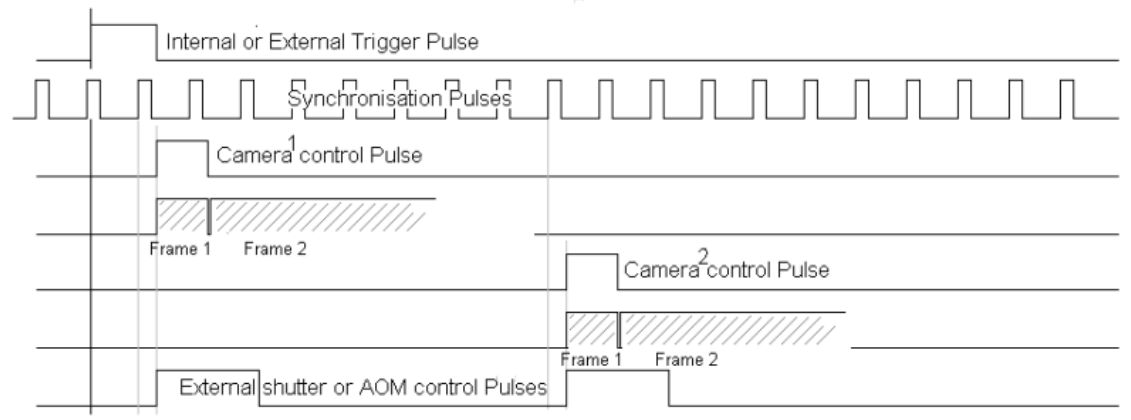

Figure 3: Timing diagram.

An OFS TC412 synchronizer interfaces to the host PC through one of the COM ports and this is used by the system software for configuring the synchronization parameters as well as reading back the period of the synchronization signal.

The duration of the camera control pulses and the AOM control pulses are derived directly from the measured scanner frequency. The delay from the internal or external trigger to the camera or AOM control pulses also depends on the selected delay between camera acquisitions. The time separation between image pairs captured by camera 1 and 2, may be selected as an integer number of scan periods.

The external trigger input to the synchronizer may be used when an acquisition event is to be triggered by some external input, coming for example from a wave maker. Internal triggering is also possible where a sequence of acquisitions may be initiated at a range of frequencies up to the maximum frame rate of the camera ( $\leq 8$ frames/second).

\subsubsection{Alignment of the cameras}

The two cameras are set up with the same field of view by simply positioning them side by side and rotated by an angle of $4.4^{\circ}$, so they both view the same region from slightly different perspectives. 
The motion to be studied with the acceleration system is strongly two-dimensional. Thus the images recorded at or near to $90^{\circ}$ to the plane of the light sheet give closely representative values of the in-plane components of velocity. For simplicity, and to maximize the effectiveness of the available light a side by side camera arrangement is used. Alternatively one could use a beam splitter in front of the cameras so that they view exactly the same region, but this would also reduce the light intensity on each image by $50 \%$. Furthermore the two-camera system is easily extendable to stereoscopic PIV for 3D measurements.

Initially the two cameras are orientated so that the two fields of view align visually. This is done by viewing images of registration marks on a perspex sheet placed in the tank at the plane of the light sheet. The cameras are first oriented perpendicular to the wall of the tank by focusing the cameras on the glass outer surface of the tank. The orientation of the cameras is then adjusted so that the image of each camera reflected on the tank wall is exactly in the centre of the respective images. The focus of the cameras is then adjusted back to the plane of the light sheet and each camera is tilted towards each other in the horizontal plane by the same amount until the images are roughly aligned according to the registration marks. The perspex sheet is then removed and the water in the tank seeded. The camera orientation is then fine-tuned by cross-correlating the base image from camera 1 with the base image of camera 2 in the same way as standard cross-correlation is used for measuring particle displacements. The vector maps produced in this way give a direct measure of the misalignment. Using on-line cross-correlation analysis alignment can be performed quite quickly and to within 2-3 pixels across the whole field of view.

Acceleration is derived from the difference between velocity measurements made from two separate and slightly different perspectives, at different time instants. It is therefore important to consider what effect the difference in perspective has on the acceleration measurements in the case of this application.

A test was carried out recording the velocity field due to the waves we discussed in section 3. But instead of triggering the cameras with a small time difference between them the cameras were triggered simultaneously. The images thus acquired were then analyzed and the vector fields compared. Because the cameras were triggered simultaneously the difference between the two vector-maps give a measure of the effect of viewing the flow from slightly different perspectives. This effect could have been estimated from geometrical considerations but this direct method also accounts for other factors such as viewing through the wave tank walls and lens distortion.

For the $4.4^{\circ}$ camera separation, used for acceleration measurements, the difference between the instantaneous vector-field measured from camera 1 and camera 2 is presented in table 1. For comparison, an angle of 8 degrees is also checked. As a reference the table also includes the mean of the displacement field due to the waves, $\left|\mathbf{d}_{\mathbf{m}}\right|=\sqrt{d_{x}^{2}+d_{y}^{2}}$. We observe that the error introduced by viewing the flow from slightly different angles is $1.9-2.6 \%$ relative to $\left|\mathrm{d}_{\mathbf{m}}\right|$. 


\begin{tabular}{|c|c|c|c|c|}
\hline Angle & Minimum & Mean & Maximum & $\left|\mathbf{d}_{\mathbf{m}}\right|$ \\
\hline $4.4^{\circ}$ & 0.00224 pixels & 0.13 pixels & 0.45 pixels & 6.90 pixels \\
\hline $8.0^{\circ}$ & 0.0056 pixels & 0.18 pixels & 0.65 pixels & 7.03 pixels \\
\hline
\end{tabular}

Table 1: Difference between vector fields as measured by two cameras.

\subsection{Acquisition and Analysis Software}

Both the cameras and the synchronizer are interfaced to the host $\mathrm{PC}$ and are controlled from a single software package (VidPIV3-Rowan, Optical Flow Systems 1999). This is a complete control and analysis software tool, including a state of the art graphical user interface.

On setting up the equipment for use in an experiment the cameras are aligned to view the desired region of flow. Then they are calibrated by generating a mapping from an acquired image of a grid with known points on it and referenced to a convenient origin. Acquired images are initially processed using cross-correlation on a grid specified in real units using the image mapping to transpose to pixel coordinates. The vector maps generated in this way are filtered using both global and local filtering and then interpolated.

Tests have also been performed with another PIV software (Sveen, 1999), finding excellent agreement with both VidPIV measurements and theory (results not shown).

\section{The experiments}

We now apply the extended PIV system to measurements of velocities and accelerations due to periodic waves propagating in a wave tank. A complementary theoretical description of the waves provides a comparison with the measurements. The purpose is to test the PIV system and judge the accuracy of the wave experiments under realistic and controllable conditions in the laboratory. The wave tank is $24.6 \mathrm{~m}$ long, $0.5 \mathrm{~m}$ wide and filled with water to a depth of $0.6 \mathrm{~m}$. In one end of the tank there is a hydraulic piston wave maker with movements controlled by a computer. At the other end there is an absorbing beach to damp the waves. The position of the cameras is $12.5 \mathrm{~m}$ from the wave maker. The measurements are terminated before any small reflected wave from the beach appear at the recording position. The wave tank and the motion of the wave maker are very precise. The wave generation in the tank has previously been examined in detail: for Stokes waves (Huseby and Grue, 1999) and for focusing wave groups (Jensen and Grue, 1999) finding excellent agreement between experiments and theoretical models.

\subsection{The incoming waves}

A sinusoidal motion of the wave maker with frequency $\omega$ generates a wave train which has a transient leading part followed by waves which are periodic. We pay attention to the periodic part of the wave train where the waves are characterized by the wave amplitude $a$, the frequency $\omega$, the wavenumber $k$ and the corresponding wave length $\lambda$. Most of the runs 
are performed with a wave length comparable to the depth $h$ of the water. This means that the waves to a good approximation are deep water waves. The surface elevation $\eta$ of the periodic waves may be described by

$$
\eta=a \cos (k x-\omega t)+a^{(2)} \cos 2(k x-\omega t)+a^{(3)} \cos 3(k x-\omega t)+\ldots
$$

including the locked higher harmonic components with amplitudes $a^{(n)}(n>1)$ accompanying the fundamental mode. In (1) the horizontal $x$-axis along the length-direction of the wave tank and the time $t$ are introduced. The surface elevation is recorded by wire gauges at several places in the wave tank to document the incoming wave field. The wave amplitude is determined from the fundamental harmonic oscillation of $\eta$. The measurements of the higher harmonic waves follow Grue (1992) and show that $a^{(2)} \simeq(1 / 2) a^{2} k$, $a^{(3)} \simeq(3 / 8) a^{3} k^{2}$ and $a^{(n)} \simeq 0$ for $n>3$ and $a k<0.2$. This means that the incoming waves may be regarded as pure Stokes waves and that the fluid motion to a good approximation may be described by the velocity potential

$$
\phi=\frac{a g}{\omega} \exp (k y) \sin (k x-\omega t)+\mathcal{O}\left(a^{4}\right)
$$

In $(2), \omega^{2}=g k\left(1+a^{2} k^{2}\right), g$ denotes the acceleration due to gravity, and the vertical $y$-axis is pointing upwards, with $y=0$ in the mean free surface. The relation (2) is a simple high-order approximation of the velocity potential, see e.g. Newman (1977). We note that small parasitic second harmonic waves are generated at the wave maker propagating at half the speed of the wave field (1)-(2). These waves arrive at a late time at the recording position, and we terminate the measurements before the parasitic waves appear.

Some of the experiments are performed when the effect of a finite water depth is essential. In this case the fluid motion is governed by the potential

$$
\phi=\frac{a g}{\omega} \frac{\cosh k(y+h)}{\cosh k h} \sin (k x-\omega t)+\mathcal{O}\left(a^{2}\right),
$$

where $\omega^{2}=g k \tanh k h\left(1+f(k h) \cdot\left(a^{2} k^{2}\right)\right)$ and $f(k h)$ is a function of $k h$, given in e.g. Mei $(1989, \S 12.3)$.

\subsection{Recording the images and filtering}

As previously noted, sequences of two image pairs are captured for the subsequent analysis of the velocity and acceleration fields of the waves. Each sequence is triggered by the computer that controls the wave maker. The triggering is very accurate and has a variability of less than the scanner period of the PIV-system (set to $12 \mathrm{~ms}$ in most of the experiments). This level of precision is important since several repetitions with the same wave conditions are performed, capturing different phases of the wave. The water in the tank is seeded with conifer pollen with diameter approximately $70 \mu \mathrm{m}$. The field of view is $23 \mathrm{~cm} \times 18 \mathrm{~cm}$ in all the runs. In most experiments an interrogation window of $32 \times 32$ pixels with an overlap of $50 \%$ is used. In some experiments the interrogation window was 
$64 \times 64$ pixels (results not shown). When the cross-correlation analysis is finished the raw velocity vectors are validated with a global window velocity filter that allows selection of velocity limits $V_{x} \min , V_{x} \max , V_{y} \min$ and $V_{y} \max$. The filter creates a scatter plot and shows the distribution of velocities in the vector map. From this plot typical values of the upper and the lower bounds of the velocity are chosen. This global filter will typically not identify outliers that are of the same magnitude as the surrounding flow, and therefore we also apply a local median filter. This filter removes vectors that fall outside a limit $\mathbf{V}_{\text {lim }}$ defined by

$$
\mathbf{V}_{\text {lim }}=\mathbf{V}_{\text {median }} \pm F \times \mathbf{V}_{\text {std }}
$$

where $F=1.5$ and $\mathbf{V}_{\text {median }}$ and $\mathbf{V}_{\text {std }}$ is the median and standard deviation, respectively, of the eight surrounding neighbors to a vector $\mathbf{V}(i, j)$. Finally identified outliers are interpolated by a $3 \times 3$ kernel with a weighted mean technique, following Wang, Barnes and Gray (1996).

\section{Results}

Two different wave frequencies are used to obtain the results shown below. Experiments with other frequencies are also performed obtaining similar results (not shown). In the first set the wave frequency is $\omega=8.95 \mathrm{~s}^{-1}$ and the amplitude measured by wire gauges is $a=2.05 \mathrm{~cm}$. The wavenumber estimated from the dispersion relation is $k=7.95 \mathrm{~m}^{-1}$ giving $\lambda=0.79 \mathrm{~m}$ and a wave slope of $a k=0.16$. The magnitudes of the fluid velocity and the acceleration are $0.17 \mathrm{~ms}^{-1}$ and $1.6 \mathrm{~ms}^{-2}$, respectively, at the mean free surface.

The output of the PIV evaluations are the two velocity fields $\mathbf{v}\left(\mathbf{x}, t_{1}\right)$ and $\mathbf{v}\left(\mathbf{x}, t_{2}\right)$ at the two time instants $t_{1}$ and $t_{2}$, respectively. In the first set of experiments the time separation between images 1 and 2 and between images 3 and 4 is $12 \mathrm{~ms}$ while $t_{2}-t_{1}$ is $60 \mathrm{~ms}$. Examples of $\mathbf{v}\left(\mathrm{x}, t_{1}\right)$ and $\mathbf{v}\left(\mathrm{x}, t_{2}\right)$ are visualized in figure 4 . We note that the velocity field at time $t_{1}$ contains some spurious vectors near the surface of the fluid. This is because the second image of camera 1 (image 2 in the sequence) contains the images 3 and 4 , since the shutter of camera 1 is open during the exposure of camera 2. The result is that the free surface in image 2 becomes relatively thick, and extraction of velocity vectors for $\mathbf{v}\left(\mathbf{x}, t_{1}\right)$ close to the free surface becomes more uncertain than inside the fluid domain. The fact that image 2 contains images 3 and 4 also means that the time separation $t_{2}-t_{1}$ should be much greater than the scan period, to avoid correlation between image 1 and images $3-4$.

The acceleration field $\partial \mathbf{v} / \partial t$ may be estimated by evaluating

$$
\frac{\mathbf{v}\left(\mathrm{x}, t_{2}\right)-\mathrm{v}\left(\mathrm{x}, t_{1}\right)}{t_{2}-t_{1}}
$$

Figure 5 shows an example of the acceleration field as calculated from the velocity fields shown in figure 4.

The measured velocity and acceleration fields may be compared with the corresponding fields due to the theoretical Stokes waves determined by (2). The latter predicts an 
exponential decay in the vertical direction according to $\exp (k y)$ of the velocities and the accelerations, with $k$ determined from the dispersion relation. It is tempting to fit the measured quantities with this exponential profile. Results obtained by a least squares minimization show that the measurements are very close to the fitted curves. Examples are displayed in figure 6 .

For further comparison of the experiments with the theory we may exploit that the magnitude of $\mathbf{v}$ and $\partial \mathbf{v} / \partial t$ of the Stokes waves (2) are independent of the horizontal coordinate $(x)$. We thus derive

$$
\begin{array}{r}
\alpha \equiv \frac{\omega|\mathbf{v}|}{a g k}=\frac{\omega}{a g k}\left[u^{2}+v^{2}\right]^{\frac{1}{2}}=\exp (k y), \\
\dot{\alpha} \equiv \frac{|\partial \mathbf{v} / \partial t|}{a g k}=\frac{1}{a g k}\left[u_{t}^{2}+v_{t}^{2}\right]^{\frac{1}{2}}=\exp (k y),
\end{array}
$$

where $(u, v)=\mathbf{v}$ and $\left(u_{t}, v_{t}\right)=\partial \mathrm{v} / \partial t$. We may check how close the measured $\alpha$ and $\dot{\alpha}$ are to the theoretical counterpart $\exp (k y)$. In figure 7 we have plotted $\alpha$ and $\dot{\alpha}$ due to the measurements in figures $5-6$ as function of the vertical coordinate $(y)$. Four horizontal positions are selected: $x / h=-0.15,-0.5,0.5,0.15$. We observe that the measurements are very close to the theoretical prediction for every abscissa. The results in figure 7 for $x / h=0.05,0.15$ are particularly good.

Repeated tests are performed capturing different phases of the waves. The measured results always exhibit velocity and acceleration profiles close to the theory (2), (6), (7) with some minor scatter. We note that the phase of the wave does not contribute to $\alpha$ and $\dot{\alpha}$, according to theory. This means that typical scatter of the measurements may be visualized by comparing these quantities for several columns of the velocity and acceleration maps. Results in figure 8 show $\alpha$ and $\dot{\alpha}$ for 20-25 columns of figures 5-6 near the crest of the wave. The experimental results fit well with the theoretical profile $\exp (k y)$ apart from some minor scatter. The scatter is somewhat larger for the accelerations than for the velocities.

To quantify the scatter we evaluate the vertical average of the row-wise standard deviation of $\alpha$ and $\dot{\alpha}$. More specifically we evaluate

$$
\sigma=\frac{1}{M} \sum_{m=1}^{M}\left(\frac{1}{N} \sum_{n=1}^{N}(V(m, n)-\bar{V}(m))^{2}\right)^{1 / 2}
$$

where $V$ is either $\alpha$ or $\dot{\alpha}, M$ and $N$ denote the number rows and columns of the velocity and acceleration maps, respectively, and $\bar{V}$ the row-wise mean given by

$$
\bar{V}(m)=\frac{1}{N} \sum_{n=1}^{N} V(m, n) .
$$

The value of $\sigma$ determined by (8) is $0.6 \%$ (relative to unity) for the velocities and $2 \%$ for the accelerations shown in figure 5. These results are due to 40 columns. Obviously, eventual small variations in the experimental wave field contribute to these digits. 
Experiments are also performed with a smaller wave amplitude of $a=1.2 \mathrm{~cm}$ with $a k=0.09$ keeping the wave frequency $\omega=8.95 \mathrm{~s}^{-1}$. In this case typical velocities and accelerations at the free surface are $0.11 \mathrm{~ms}^{-1}$ and $0.96 \mathrm{~ms}^{-2}$, respectively. Results for 40 columns of the velocities and accelerations are shown in figure 9. The results fit quite well with the theoretical curve $\exp (k y)$. There is, however, a larger scatter in the results for the smaller wave amplitude than for the larger. The value of $\sigma$ determined by ( 8 ) is $3 \%$ for the velocities and $7 \%$ for the accelerations shown in figure 9. These results illustrate that the relative accuracy becomes poorer when the wave amplitude and thereby the fluid velocity is reduced. Conversely, the relative accuracy is improved when the wave amplitude is increased. This also illustrates that high accuracy may be achieved by appropriately choosing the size of the field of view.

We have also done some experiments with relatively long waves, with $\omega=3.77 \mathrm{~s}^{-1}$ and $a=7.5 \mathrm{~cm}(a k=0.14)$. In this case the magnitude of the fluid velocity at the mean free surface is $0.36 \mathrm{~ms}^{-1}$ while the fluid acceleration is $1.4 \mathrm{~ms}^{-2}$. Furthermore, $\lambda=3.46 \mathrm{~m}$ and $k h=1.09$, thus the effect of a finite depth has to be taken into account. From (3) we may derive

$$
\begin{gathered}
\beta \equiv \frac{\omega \cosh k h}{a g k}\left[\frac{u^{2}}{\cosh ^{2} k(y+h)}+\frac{v^{2}}{\sinh ^{2} k(y+h)}\right]^{\frac{1}{2}}=1, \\
\dot{\beta} \equiv \frac{\cosh k h}{a g k}\left[\frac{u_{t}^{2}}{\cosh ^{2} k(y+h)}+\frac{v_{t}^{2}}{\sinh ^{2} k(y+h)}\right]^{\frac{1}{2}}=1 .
\end{gathered}
$$

In figure 10 we have plotted the measured $\beta$ and $\dot{\beta}$. The scanner period is now 8 ms while $t_{2}-t_{1}=64 \mathrm{~ms}$. The measurements are rather close to the theory. The value of $\sigma$ due to 40 columns determined by (8) is $3 \%$ for the velocities and $7 \%$ for the accelerations.

\section{Conclusion}

An extended PIV system to measure the accelerations $\partial \mathbf{v} / \partial t$ of a velocity field $\mathbf{v}$ of a fluid is described. The system consists of two CCD cameras, a scanning laser beam and a synchronizer controlled and monitored by a computer. The laser source is a CW argon ion laser, sufficiently powerful $(10 \mathrm{~W})$ to provide light for sequences of recorded image pairs. The two high-sensitive cooled PCO Sensicam cameras have a resolution of $1280 \times 1024$ pixels with 12-bit digital output.

A high speed acousto-optic modulator is used to shut off the CW laser after two scans of the flow have been captured by the first camera. The modulator switches the beam back on for the second velocity measurement after a programmed delay. Synchronization of the cameras and beam modulator with the scanning beam system is achieved with a purpose built multi-channel synchronizer device and operated from an integrated modular tree-based acquisition and processing software system.

The system is applied to measure the accelerations due to water waves in a wave tank of standard laboratory size. It is tested for several wave parameters in the laboratory. Results 
are displayed for waves with frequency $\omega=8.95 \mathrm{~s}^{-1}$ and amplitude either $1.09 \mathrm{~cm}$ or $2.05 \mathrm{~cm}$. In another set of experiments the frequency is $\omega=3.77 \mathrm{~s}^{-1}$ and the amplitude is $7.5 \mathrm{~cm}$. In all cases the water depth is $0.6 \mathrm{~m}$. The fluid velocity and the acceleration at the position of mean free surface are in the ranges $0.11-0.36 \mathrm{~ms}^{-1}$ and $0.96-1.6 \mathrm{~ms}^{-2}$, respectively. The experiments are compared with theoretical Stokes waves. In the cases with wave frequency $\omega=8.95 \mathrm{~s}^{-1}$ the waves are deep water waves, practically speaking. The theoretical model is then valid up to the third order in the wave slope, giving an error term being less than $0.5 \%$ relative to the primary wave, for the conditions in the experiments. Thus the theory is very precise.

With the cross correlation technique used in the PIV analysis we get an absolute error of the velocity measurements which may not in practice be reduced beyond a certain value. The relative error is hence smaller for low velocity flows than for large velocity flows. This is confirmed by the experiments (figures $8-9$ ). This means that a small relative error may generally be achieved by appropriately choosing the size of the field of view. The accelerations are found to be somewhat more noisy than the velocities since they are obtained by a finite difference method. However, agreement between theory and experiment is satisfactory. The relative accuracy of the present experiments and measurements may be quantified in terms of the standard deviation due to an ensemble of measurements, determined here by (8). In the best case we find a relative standard deviation of $0.6 \%$ for the velocity measurements and $2 \%$ for the accelerations. We thus conclude that our PIV system is efficient for velocity and acceleration measurements due to gravity waves.

It may be relevant to briefly compare our measurements of accelerations in water waves with those of others. Chang and Liu (1998) measure velocities and accelerations in breaking waves. Their wave data are: frequency $\omega=6.28 \mathrm{~s}^{-1}$, amplitude approximately $15 \mathrm{~cm}$, typical velocity $2 \mathrm{~ms}^{-1}$, maximal acceleration about $10 \mathrm{~ms}^{-2}$, water depth $0.2 \mathrm{~m}$, and the measurement position is $0.35 \mathrm{~m}$ from the wave paddle. They comment that their PIV system gives rather good accuracy for the cases investigated. Another group (Jacobsen et al. 1997) has measured the velocities and accelerations due to standing waves with frequency $\omega=6.28 \mathrm{~s}^{-1}$ and amplitude approximately $6 \mathrm{~cm}$, finding good results for the velocities but rather uncertain results for the acceleration field. It may be true that the novel highresolution and high-sensitive extended PIV system outlined here, and that by Chang and Liu exploiting a different system from ours, provide significant technological improvements of previous techniques to measure accelerations in fluids. With the continuous development of camera technology, similar but more precise systems are expected to appear in the near future. We find, however, that the extended PIV system outlined here is quite suitable for investigations of various unresolved aspects of nonlinear gravity waves.

We have in this investigation considered the acceleration term $\partial \mathbf{v} / \partial t$. The complete acceleration is obtained by adding $\mathbf{v} \cdot \nabla \mathbf{v}$ which involves evaluation of $\nabla \mathbf{v}$. Fairly precise evaluation of the latter term my be obtained by numerical differentiation of the velocity field. (Examples for internal waves are found in Grue et al., 1999.) We finally note that the present two camera PIV-system is easily extendable to 3-D PIV measurements and combined PIV and Laser Induced Fluorescence measurements. 
This work was conducted under the Strategic University Programme 'General Analysis of Realistic Ocean Waves' funded by the Research Council of Norway. The technical assistance by Mr. Arve Kvalheim and Mr. Svein Vesterby is gratefully acknowledged.
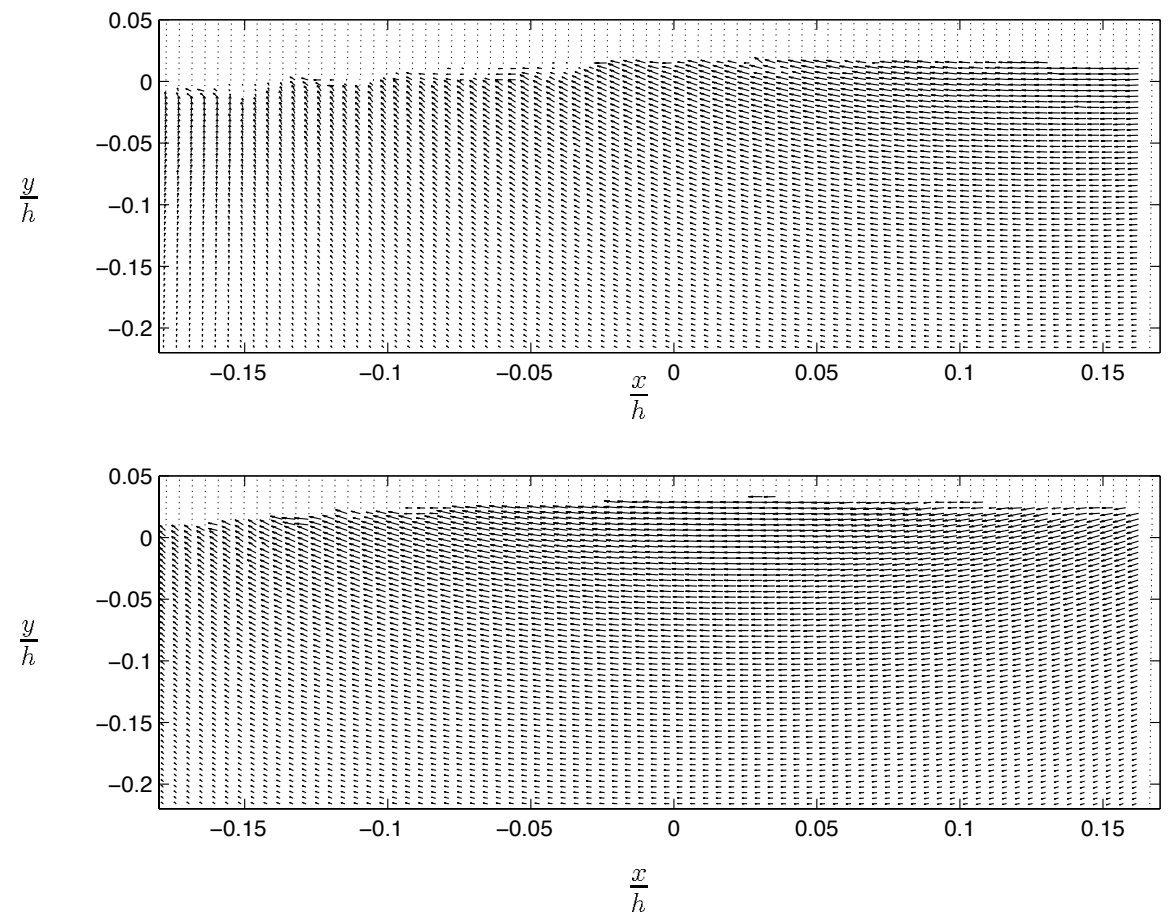

Figure 4: Velocity fields. Waves with $\omega=8.95 \mathrm{~s}^{-1}, a k=0.16$ and $k h=4.9$. Top: $\mathbf{v}\left(\mathrm{x}, t_{1}\right)$. Bottom: $\mathbf{v}\left(\mathbf{x}, t_{2}\right)$. 


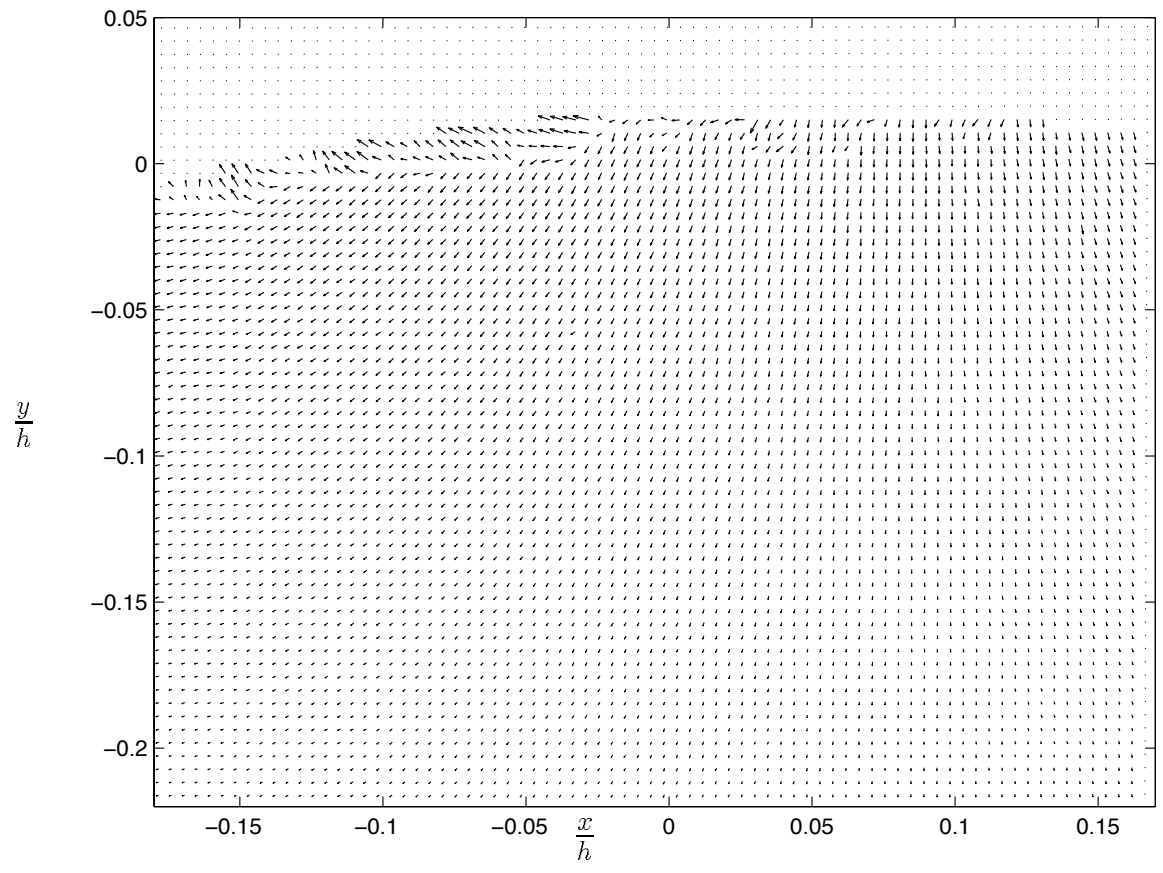

Figure 5: Acceleration field, $\frac{\mathbf{v}\left(\mathbf{x}, t_{2}\right)-\mathbf{v}\left(\mathbf{x}, t_{1}\right)}{t_{2}-t_{1}}$. 

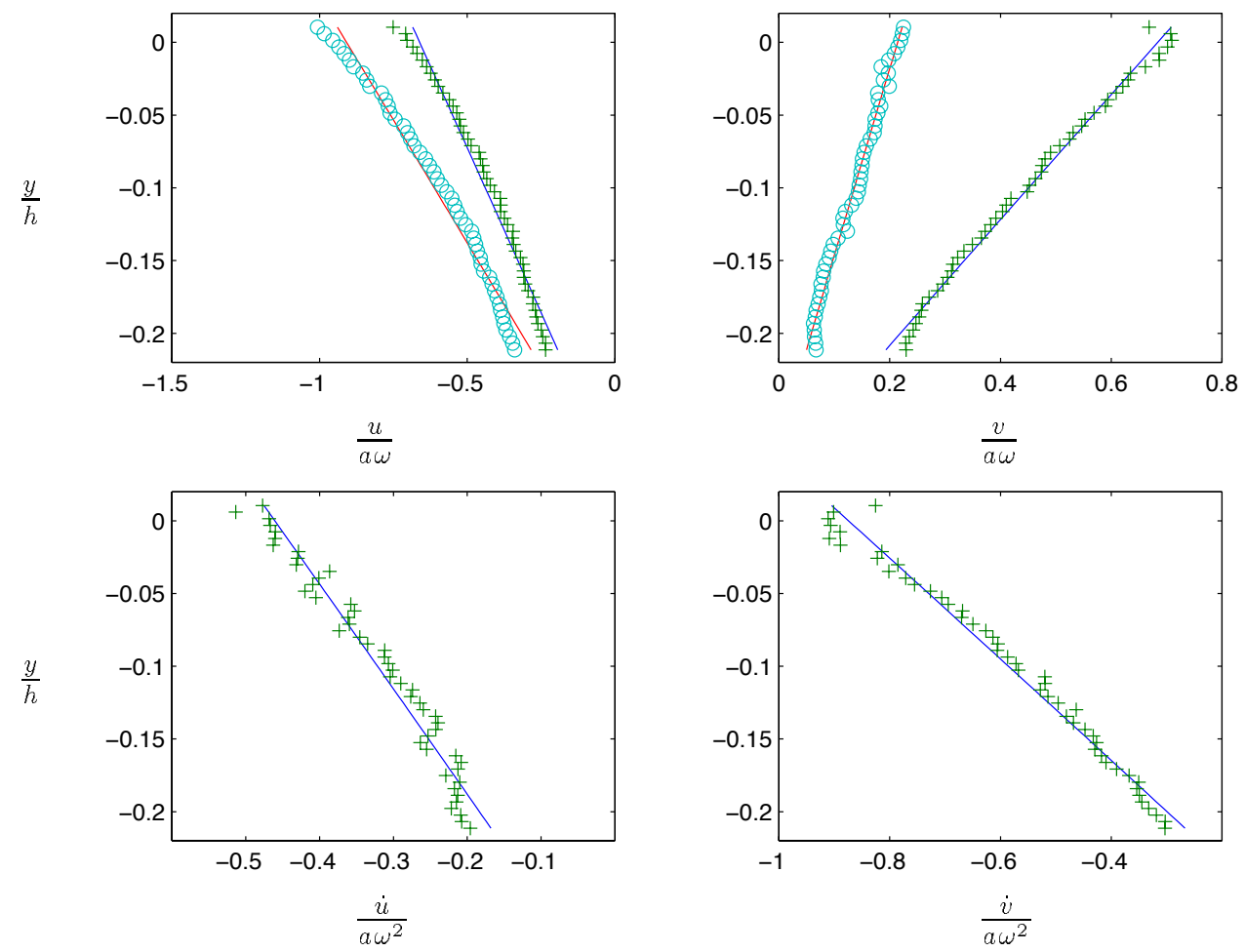

Figure 6: Velocity and acceleration measurements. $\omega=8.95 \mathrm{~s}^{-1}, a k=0.16, k h=4.9$. Solid line theory. Top left: ' + ' and 'o' experimental velocity profiles $u\left(\mathrm{x}, t_{1}\right)$ and $u\left(\mathrm{x}, t_{2}\right)$ respectively, $x$-position fixed. Top right: Same as the left figure except that this contains the vertical velocity profiles $v\left(\mathbf{x}, t_{1}\right)$ and $v\left(\mathbf{x}, t_{2}\right)$. Bottom left: ' + ' horizontal acceleration profile $\left(u\left(\mathrm{x}, t_{2}\right)-u\left(\mathrm{x}, t_{1}\right)\right) /\left(t_{2}-t_{1}\right)$. Bottom right: '+' vertical acceleration profile $\left(v\left(\mathrm{x}, t_{2}\right)-\right.$ $\left.v\left(\mathrm{x}, t_{1}\right)\right) /\left(t_{2}-t_{1}\right)$. 

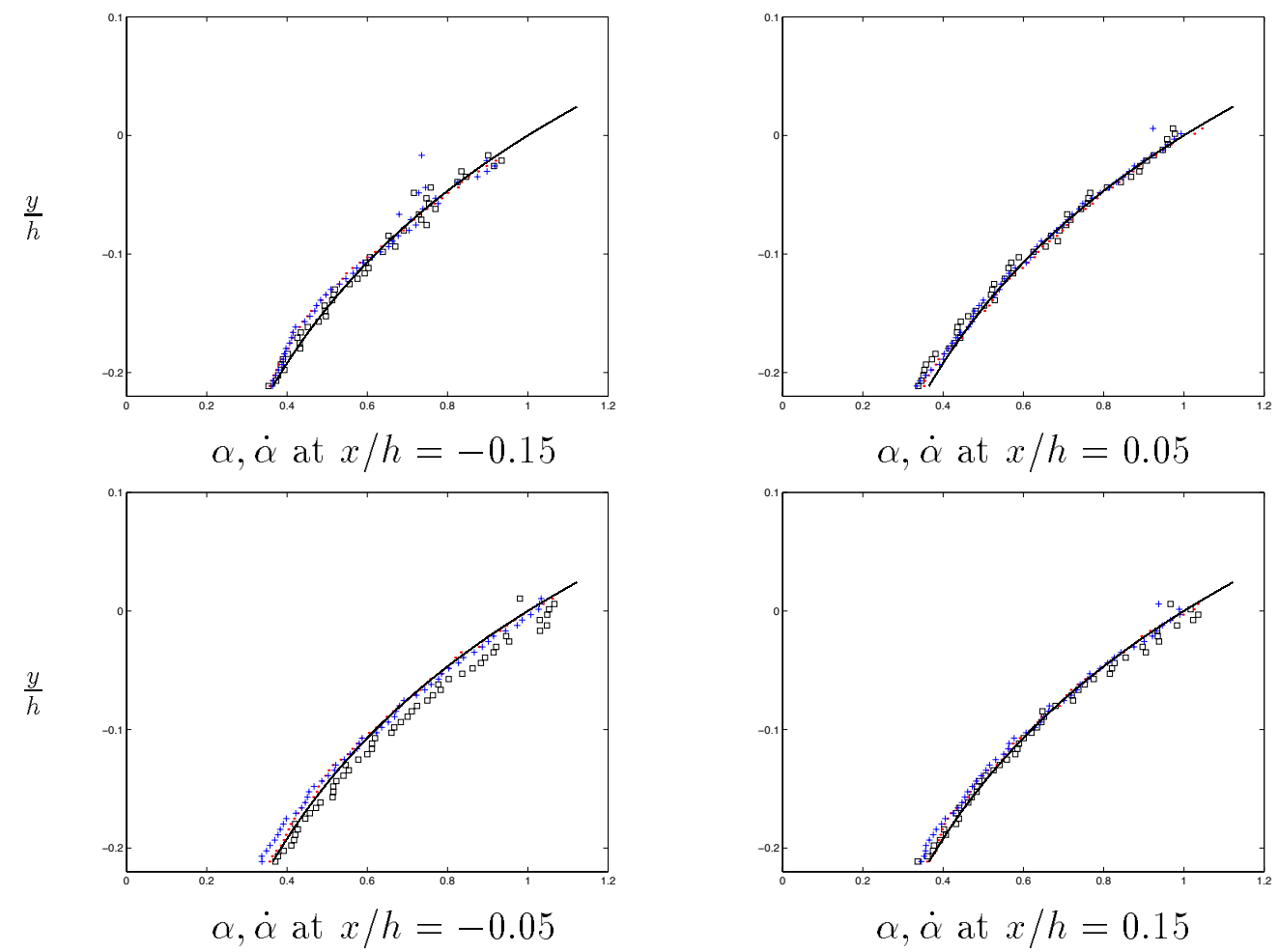

Figure 7: Velocity and acceleration measurements. $\omega=8.95 \mathrm{~s}^{-1}$, $a k=0.16, k h=4.9$. '.' $\alpha(y)$ for $t=t_{1}$, ' + ' $\alpha(y)$ for $t=t_{2}$ and squares are $\dot{\alpha}(y)$. Top left: $\alpha(y)$ and $\dot{\alpha}(y)$ for $x / h=-0.15$. Bottom left: Same as above except $x / h=-0.05$. Top right: $x / h=0.05$. Bottom right: $x / h=0.15$. $\alpha$ and $\dot{\alpha}(y)$ defined in the text. Solid line $\exp (k y)$. 


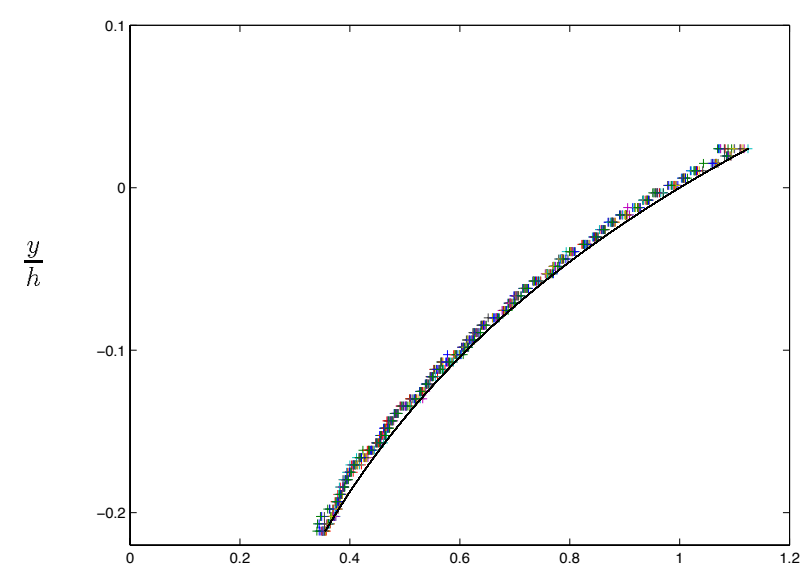

$\alpha$

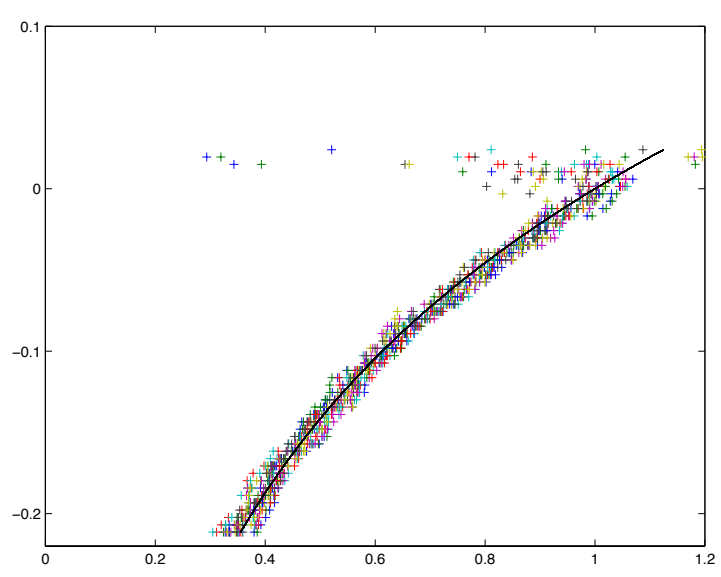

$\dot{\alpha}$

Figure 8: Velocity and acceleration measurements, $\omega=8.95 \mathrm{~s}^{-1}, a k=0.16$ and $k h=4.9$. Left: ' + ' are 20 columns of velocity measurements taken from the velocity field of the crest. Right: Same as the left except that this is acceleration measurements (25 columns). $\alpha$ and $\dot{\alpha}(y)$ defined in the text. Solid line $\exp (k y)$.
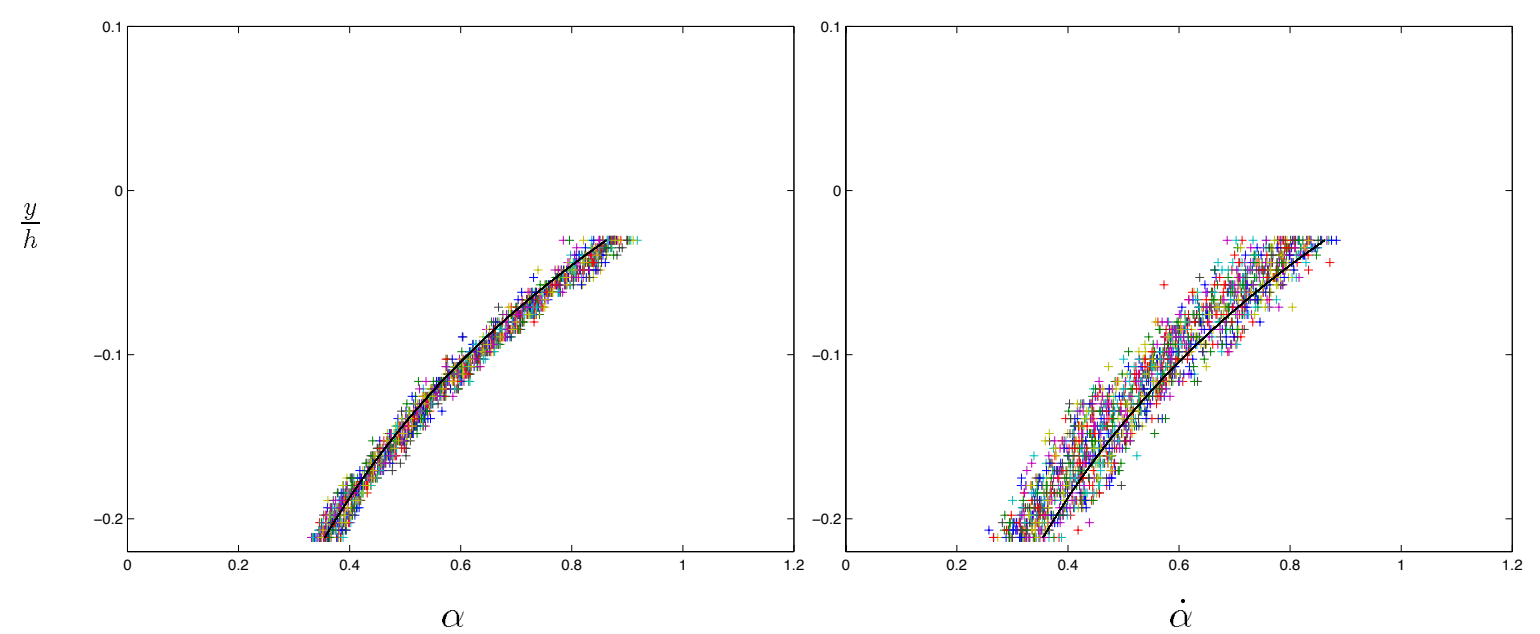

Figure 9: Same as figure 8, but $a k=0.09,40$ columns. 

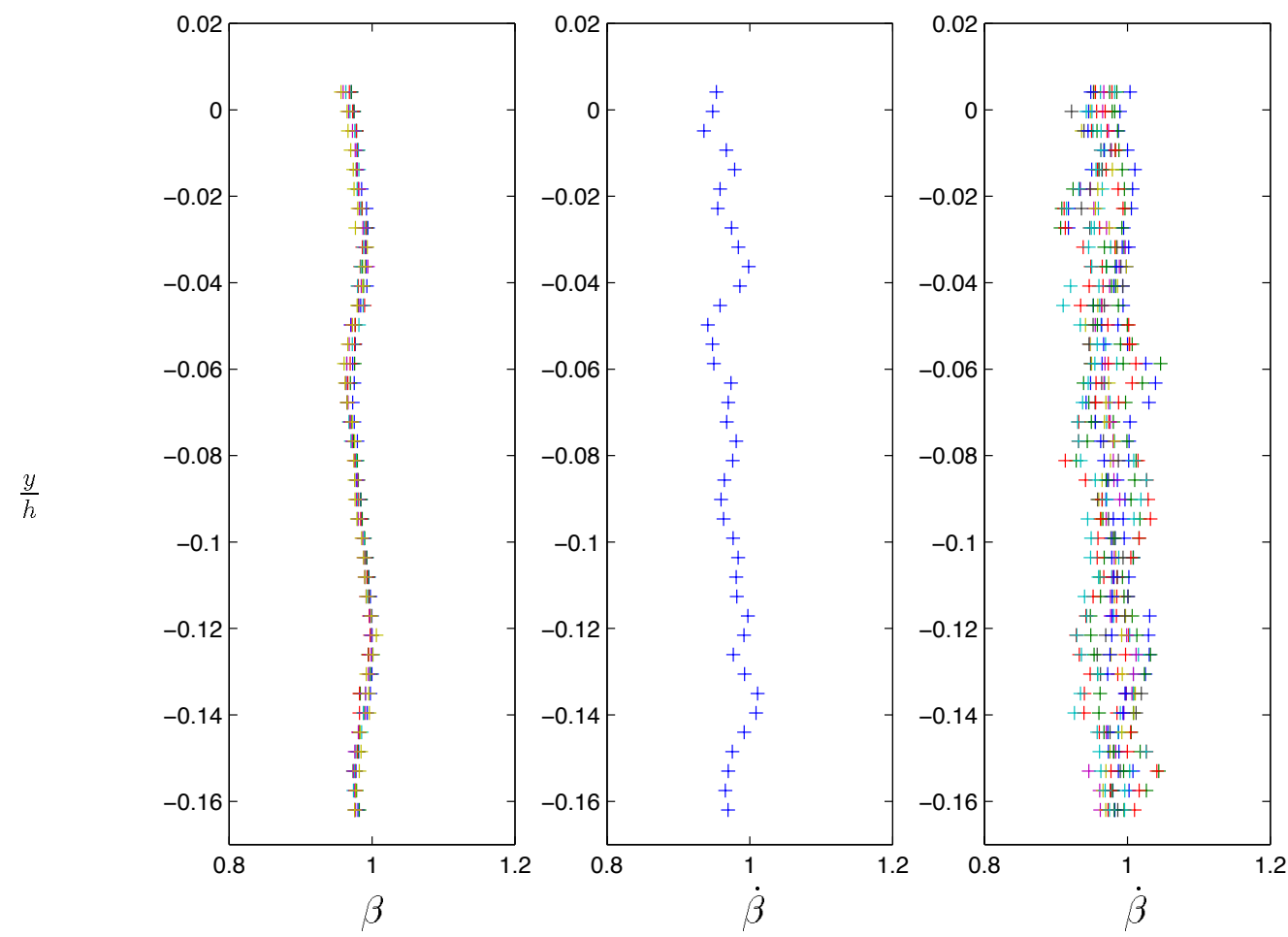

Figure 10: Velocity and acceleration measurements, $\omega=3.77 \mathrm{~s}^{-1}, a k=0.14$ and $k h=1.09$. Left: '+' are 5 columns of velocity measurements taken from the velocity field. Middle: Acceleration measurements, one column. Right: Acceleration measurements, 10 columns. $\beta$ and $\dot{\beta}$ are defined in the text. 


\section{References}

[1] K.-A. Chang \& P. L.-F Liu: "Velocity, acceleration and vorticity under breaking waves.", Physics of Fluids,10 1 : 327-329, 1998.

[2] C. Gray, C. A. Greated, D. R. McCluskey, W. J. Easson: "An analysis of the scanning beam PIV illumination system". Meas. Sci. Technol. 2: pp 717-724, 1991.

[3] J. Grue: "The nonlinear water waves at a submerged obstacle or bottom topography". J. Fluid Mech.,244, pp 455-476, 1992.

[4] J. Grue, A. Jensen, P. O. Rusaas and J. K. Sveen: "On the breaking and broadening of internal solitary waves propagating in stratified fluid". Pre-Print, Dept. of Mathematics, UiO. 1999. ISSN 0809-4403. Submitted for publication.

[5] M. Huseby and J. Grue: "An experimental investigation of higher harmonic forces on a vertical cylinder". Pre-Print, Dept. of Mathematics, UiO. 1999. ISSN 0809-4403. Submitted for publication.

[6] M. L. Jakobsen, T. P. Dewhirst and C. A. Greated: "Particle image velocimetry for predictions of acceleration fields and force within fluid flows". Meas. Sci. Technol., 8: $1502-1516,1997$.

[7] A. Jensen and J. Grue: "On the difference in the speed of gravity waves in a physical and numerical wave tank". Pre-Print, Dept. of Mathematics, UiO. 1999. ISSN 08094403. Submitted for publication.

[8] C. C. Mei: "The applied dynamics of ocean surface waves." World Scientific. 740 pp, 1989.

[9] J. N. Newman: "Marine Hydrodynamics." MIT Press. 402 pp, 1977.

[10] J. K. Sveen: "An introduction to MatPIV 1.4". Documentation and code: http://www.math.uio.no/ jks/matpiv/, 1999.

[11] "VidPIV3 Rowan User Manual”, Optical Flow Systems, 1999.

[12] J. Wang, F. Barnes and C. Gray: "A comparison between interpolation algorithms for post processing of particle image velocimetry data". proceedings of Optical Methods in Heat and Fluid Flow, City University, UK, 1996. 